\title{
Dimerization of MLL fusion proteins and FLT3 activation synergize to induce multiple-lineage leukemogenesis
}

\author{
Ryoichi Ono,,1,2 Hideaki Nakajima, ${ }^{3}$ Katsutoshi Ozaki,, ${ }^{1}$ Hidetoshi Kumagai, ${ }^{1}$ Toshiyuki Kawashima, ${ }^{3}$ \\ Tomohiko Taki, 4 Toshio Kitamura, ${ }^{3}$ Yasuhide Hayashi, ${ }^{5}$ and Tetsuya Nosaka ${ }^{1}$ \\ 1Division of Hematopoietic Factors, The Institute of Medical Science, ${ }^{2}$ Department of Pediatrics, Graduate School of Medicine, and \\ ${ }^{3}$ Division of Cellular Therapy, The Institute of Medical Science, The University of Tokyo, Tokyo, Japan. ${ }^{4}$ Department of Molecular Laboratory Medicine, \\ Kyoto Prefectural University of Medicine Graduate School of Medical Science, Kawaramachi-Hirokoji, Kyoto, Japan. \\ ${ }^{5}$ Gunma Children's Medical Center, Kitatachibana, Gunma, Japan.
}

\begin{abstract}
The mechanisms by which mixed-lineage leukemia (MLL) fusion products resulting from in utero translocations in 11q23 contribute to leukemogenesis and infant acute leukemia remain elusive. It is still controversial whether the MLL fusion protein is sufficient to induce acute leukemia without additional genetic alterations, although carcinogenesis in general is known to result from more than 1 genetic disorder accumulating during a lifetime. Here we demonstrate that the fusion partner-mediated homooligomerization of MLL-SEPT6 is essential to immortalize hematopoietic progenitors in vitro. $M L L$ SEPT6 induced myeloproliferative disease with long latency in mice, but not acute leukemia, implying that secondary genotoxic events are required to develop leukemia. We developed in vitro and in vivo model systems of leukemogenesis by MLL fusion proteins, where activated FMS-like receptor tyrosine kinase 3 (FLT3) together with MLL-SEPT6 not only transformed hematopoietic progenitors in vitro but also induced acute biphenotypic or myeloid leukemia with short latency in vivo. In these systems, MLL-ENL, another type of the fusion product that seems to act as a monomer, also induced the transformation in vitro and leukemogenesis in vivo in concert with activated FLT3. These findings show direct evidence for a multistep leukemogenesis mediated by MLL fusion proteins and may be applicable to development of direct $M L L$ fusion-targeted therapy.
\end{abstract}

\section{Introduction}

Recurrent translocations involving chromosome 11 band q23 (11q23) are frequent cytogenetic abnormalities observed in hematological malignancies, occurring in approximately $80 \%$ of infant and $10 \%$ of adult acute leukemias $(1,2)$. The mixed-lineage lenkemia (MLL) gene (also called ALL1 or HRX) has been cloned in $11 \mathrm{q} 23$ translocations, such as $\mathrm{t}(4 ; 11), \mathrm{t}(9 ; 11)$, and $\mathrm{t}(11 ; 19)(3,4)$, and more than 30 partner genes fused with $M L L$ have been identified in various types of $11 \mathrm{q} 23$ translocations (5).

$M L L$ encodes a nuclear protein characteristic of several domains with assigned activities including an $\mathrm{N}$ terminus with 3 AT-hook motifs, a CXXC domain, 4 cysteine-rich zinc fingers, a transactivation domain, and a highly conserved C-terminal $\mathrm{Su}(\mathrm{var}) 3-9$, Enhancer of zeste, and Trithorax (SET) domain with histone methyltransferase activity $(6,7)$. Recent studies demonstrated that MLL is cleaved by Taspase 1 , generating 2 fragments that heterodimerize to stabilize the complex (8) and assemble in a chromatin-modifying supercomplex (7).

Nonstandard abbreviations used: AML, acute myeloid leukemia; ENL, eleven nineteen leukemia; FLT3, FMS-like receptor tyrosine kinase 3; 5-FU, 5-fluorouracil; hER, human estrogen receptor; HOX, homeobox gene; IRES-EGFP, internal ribosomal entry site-enhanced GFP; ITD, internal tandem duplication; LBD, ligand-binding domain; Meis1, myeloid ecotropic viral integration site 1; MEs, MLL-ENLs; MLL, mixed-lineage leukemia; 5'-MLL, the portion of MLL within MLL-SEPT6; MLL-ENLs, MLL-ENL short form; MPD, myeloproliferative disease; MS6, MLL-SEPT6; 4-OHT, 4-hydroxy-tamoxifen; SET, Su(var)3-9, Enhancer of zeste, and Trithorax.

Conflict of interest: The authors have declared that no conflict of interest exists.

Citation for this article: J. Clin. Invest. 115:919-929 (2005)

doi:10.1172/JCI200522725.
Some important insights into the leukemogenesis by MLL fusion proteins have been obtained $(9,10)$. MLL fusion genes express in-frame chimeric proteins that harbor the $\mathrm{N}$-terminal amino acids of MLL and C-terminal amino acids of each partner protein and reside in the nucleus. Fusion of MLL with a translocation partner, not simple truncation of MLL, leads to development of leukemias in experimental models. Despite sharing little sequence homology to each other among various MLL fusion partners, the oncogenic activity of MLL fusion proteins has been demonstrated to arise from at least 2 distinct common mechanisms (10). One mechanism leads to the aberrant activation of target genes including homeobox genes (HOXs) by fusion of MLL with transcriptional activation domains within translocation partners such as eleven nineteen leukemia (ENL) (9). The other leads to the similar aberrant activation by dimerization of MLL fusion proteins through oligomerization domains within various translocation partners $(11,12)$. However, it remains unclear whether fusion with all MLL translocation partners leads to development of leukemia through either mechanism. Furthermore, various experimental models in vivo using biotechnological methods such as knock-in (13) and an inducible Cre-lox translocation system (14) as well as retroviral transduction did not reproduce acute leukemia with as short a latency as expected from clinical features of infant leukemia. Therefore, some secondary genotoxic events acquired by the disruptive effects of MLL fusion genes on DNA repair and/or cell-cycle regulation might play an essential role in leukemogenesis by MLL fusion proteins (15); however, this hypothesis has not been thoroughly investigated. 
A
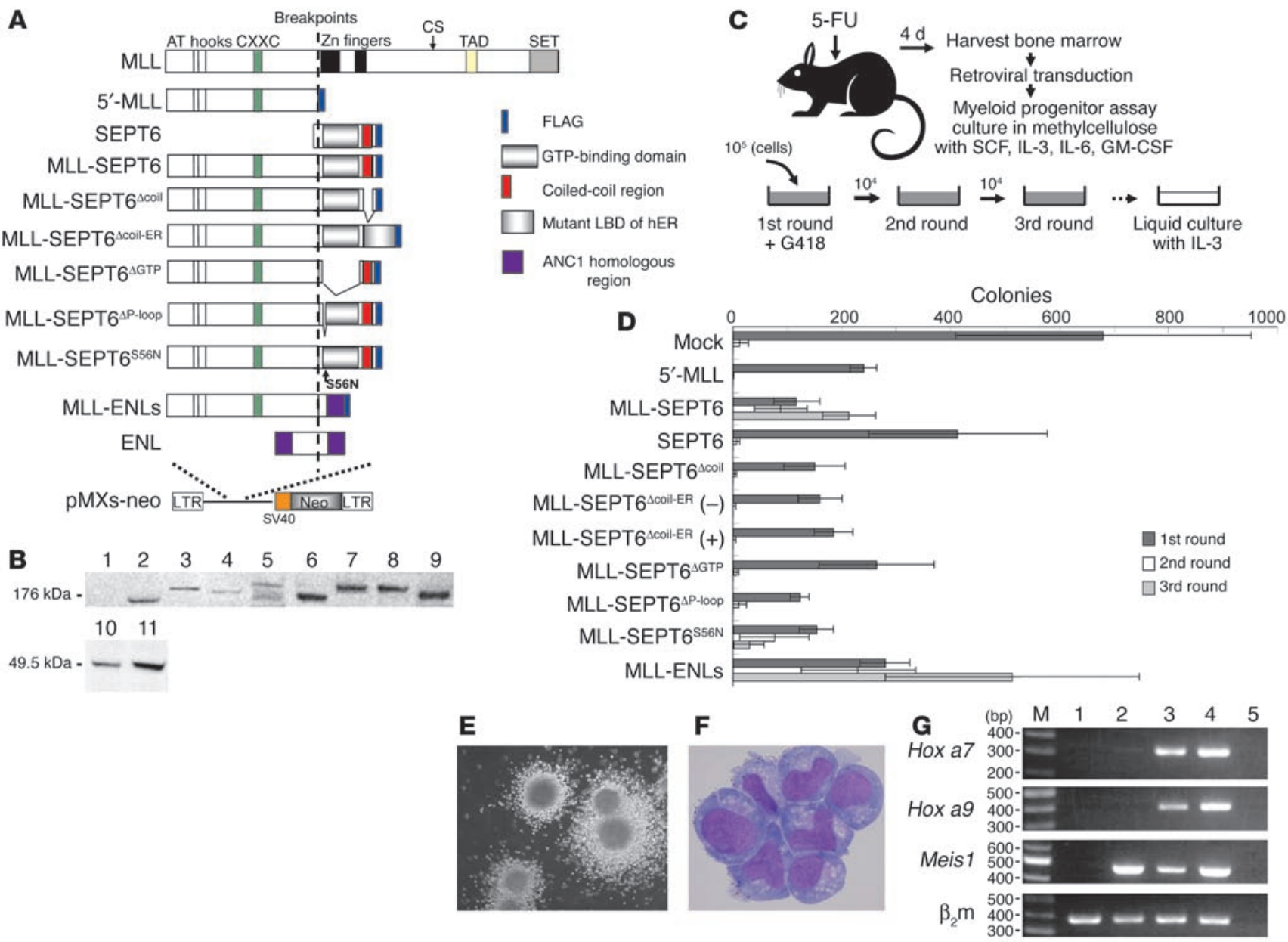

Figure 1

Immortalization of murine hematopoietic progenitors by MLL-SEPT6 fusion protein via aberrant expression of Hox genes. (A) Schematic representation of the retroviral constructions used. CXXC, CXXC domain; Zn fingers, zinc fingers; CS, cleavage sites; TAD, transactivation domain; SET, SET domain. (B) Western blot analysis of proteins extracted from PlatE cells transfected with the constructs shown in A, after immunoprecipitation using the anti-MLL Ab (lanes 1-9). Each lysate was blotted with the anti-FLAG Ab (lanes 1-9) or anti-SEPT6 Ab (lanes 10 and 11). Endogenous expression of SEPT6 was detected in lane 10. Lane 1, mock; lane 2, 5'-MLL; lane 3, MLL-SEPT6; lane 4, MLL-SEPT6 ${ }^{\Delta c o i l}$; lane 5, MLL-SEPT6 ${ }^{\Delta \text { coil-ER; lane 6, MLL-SEPT6 }}{ }^{\Delta G T P}$; lane 7, MLL-SEPT6 ${ }^{\triangle P-l o o p}$; lane 8, MLL-SEPT6S56N; lane 9, MLL-ENLs; lane 10, pMXs-neo alone (endogenous SEPT6); lane 11, SEPT6. (C) Experimental strategy for myeloid immortalization assay. (D) Myeloid immortalization assay using the constructs shown in A. Lanes for MLL-SEPT6 ${ }^{\Delta c o i l-E R}$ indicate the presence $(+)$ or absence $(-)$ of 4-OHT. The bar graph shows numbers of colonies obtained after each round of replating in methylcellulose (average \pm SD). (E and F) Typical morphology of the colonies generated by MLL-SEPT6 (E), and the cells constituting these colonies (F). Original magnification, $\times 40(\mathbf{E}), \times 400$ (F). (G) Expression of Hox a7, Hox a9, and Meis 1 by RT-PCR in the cells from third-round cultures. $\beta_{2} \mathrm{~m}$ was used as an internal standard. M, 100-bp DNA ladder (New England Biolabs Inc.); lane 1, control (Ba/F3 with IL-3) cells; lane 2, mock; lane 3, MLL-SEPT6; lane 4, MLL-ENLs; lane 5, negative control.

We identified the SEPT6/SEPTIN6 gene on chromosome Xq24 as a novel fusion partner of $M L L$ in infant acute myeloid leukemia (AML) (16). SEPT6 contains a short coiled-coil region at the $\mathrm{C}$ terminus, and a GTP-binding domain characteristic of the septin family proteins that are important for cytokinesis through polymerization $(17,18)$. Besides SEPT6, 3 of 12 human SEPTINs identified to date, SEPT5/CDCREL1, SEPT9/AF17q25/ $M S F$, and FLJ10849, have been shown to be fusion partners of MLL (19-22). MLL-SEPT6 contains almost the entire SEPT6, similar to other MLL-SEPTINs. Interestingly, according to previous case reports of $8 M L L$-SEPT6 patients (23-26), MLL-SEPT6 is involved preferentially in infant AML, while the other $M L L$ SEPTINs remain unclear.
In the present study, we first analyzed the oncogenic potential of MLL-SEPT6 in vitro by colony-formation assay after retroviral transduction and found that MLL-SEPT6 immortalized murine hematopoietic progenitors with aberrant expression of Hox a7 and Hox 99 as it did in a previously reported case of $M L L-E N L$ (9). Mutation analysis of the portion of SEPT 6 demonstrated that the fusion partner-mediated homo-oligomerization of MLL-SEPT6 is essential for leukemogenesis. We next analyzed the leukemogenic potential of MLL-SEPT6 in vivo using retroviral transduction followed by bone marrow transplantation. MLL-SEPT6 induced no acute leukemia but, rather, lethal myeloproliferative disease (MPD) with prolonged latency in vivo, far from clinical features. To examine the effect of a putative secondary genotoxic stress on 
the MLL-SEPT6-transduced cells that proliferated with IL-3, the effect of additional expression of FMS-like receptor tyrosine kinase 3 (FLT3) internal tandem duplication (ITD) was tested in vitro. Transduction of FLT3-ITD enabled the MLL-SEPT6-transduced cells to grow and expand in the absence of IL-3, and simultaneous expression of MLL-SEPT6 and FLT3-ITD led to AML with short latency in vivo. Furthermore, $M L L-E N L$, which requires no dimerization, also induces the same transformation in vitro and AML in vivo in concert with FLT3-ITD. This in vitro transformation system and in vivo leukemic development system using murine hematopoietic progenitors through the MLL fusion protein together with the secondary genotoxic stress are useful complementary models to elucidate the molecular mechanism of leukemogenesis by MLL fusion proteins.

\section{Results}

MLL-SEPT6 immortalizes murine hematopoietic progenitors with aberrant expression of Hox a7 and Hox a 9 in vitro as MLL-ENL does. To examine the oncogenic potential of MLL-SEPT6 in vitro, MLL-SEPT6, the portion of MLL within MLL-SEPT6 (5'-MLL), SEPT6, and MLLENL short form (MLL-ENLs) (9) were subcloned into pMXs-neo (27) (Figure 1A). After confirmation, by Western blot analysis, of expression of these genes in packaging cells, the enriched murine hematopoietic progenitors were analyzed by the myeloid immortalization assay using retroviruses harboring these genes or harboring none of them (named mock) (Figure 1, B and C). Initial plating showed similar morphologies and reflected transduction efficiencies. In serial replating, MLL-SEPT6-transduced cells yielded and maintained increasing numbers of compact colonies similar to those generated by MLL-ENLs, although the cells transduced with mock, 5'-MLL, or SEPT6 rapidly failed to form colonies (Figure $1 \mathrm{E})$. Wright-Giemsa-stained cytospin preparations of the cells constituting these compact colonies showed features consistent with myelomonocytic blasts (Figure 1F). Furthermore, RT-PCR of total RNA extracted from the colonies transduced with mock, MLL-
SEPT6, and MLL-ENLs at the third round using primers Hoxa7S and Hoxa7AS, or Hoxa9S and Hoxa9AS, revealed that expression of both Hox a7 and Hox a9 was upregulated in MLL-SEPT6transduced cells, as in MLL-ENLs (9), but not in mock-transduced cells (Figure 1G). The RT-PCR using primers M1S and M1AS also generated 468-bp PCR products as expected, and sequence analysis confirmed that these products represented transcripts of the Hox cofactor myeloid ecotropic viral integration site 1 (Meis1), which was reported to be upregulated with aberrant expression profiles of Hox (11). Unexpectedly, we could not detect any significant difference of expression levels of Meis 1 among these colonies.

Single-cell suspensions obtained from the third-round colony could grow and expand in liquid culture supplemented with IL-3 for more than 10 months but failed to survive without IL-3. These immortalized cells expressed Gr-1, CD11b, and c-kit but were negative for Sca-1, B220, CD3, and Ter119 as determined by fluorescence-activated cell sorting (FACS; BD Biosciences) analysis (Figure $2 \mathrm{~B}$ and data not shown) and contained oligoclonal bands of proviral integration sites, as well as a single band of the full-length proviral DNA, by Southern blot analysis using the Neo probe (Figure 2, A and C). Expression of MLL-SEPT6 chimeric transcripts could be detected by RT-PCR of total RNA extracted from the immortalized cells (Figure 2D), while MLL-SEPT6 fusion proteins were hardly detectable in the lysate of the immortalized cells (data not shown). This showed the toxicity of high expression levels, as previously reported in the literature on leukemogenesis by MLL fusion proteins using retroviral transduction (9). Taken together, these results demonstrated that MLL-SEPT6 directly immortalizes murine hematopoietic cells via both the block of differentiation and the enhancement of self-renewal in vitro, with aberrant upregulation of Hox a7 and Hox a9, as MLL-ENL does.

Myeloid immortalization in vitro by MLL-SEPT6 requires dimerization of MLL fusion proteins through both its GTP-binding domain and its coiledcoil region. Because of common structural characteristics in 4 kinds of MLL-SEPTIN fusion proteins, we examined whether both a GTP-

\section{Figure 2}

Characterization of the cells immortalized by MLLSEPT6. (A) Schematic representation of pMXsneo-MLL-SEPT6. The restriction endonuclease sites and the Neo probe used in the Southern blot analysis are indicated by vertical arrows and a thick horizontal line, respectively. The primers used are indicated by horizontal arrows. LTR, long-terminal repeat. (B) Immunophenotype of the cells immortalized by MLL-SEPT6. Shadow profiles represent FACS staining obtained with Abs specific for the indicated cell surface antigens. Green lines represent staining obtained with isotype control Abs. (C) Southern blot analysis to detect clonality (lane 1) and integration (lane 2). Genomic DNA extracted from the cells immortalized by MLL-SEPT6 was digested with BamHI (lane 1) or Nhel (lane 2), and hybridized with the Neo probe. Oligoclonal bands of proviral integration sites and a single band of the intact proviral DNA are indicated by arrows and an arrowhead, respectively. (D) Expression of MLL-SEPT6 fusion transcripts by RT-PCR. M, 1-kb DNA ladder (New England Biolabs Inc.); lane 1, cells immortalized by MLL-SEPT6; lane 2, negative control; lane 3, positive control (pMXs-neo-MLL-SEPT6).

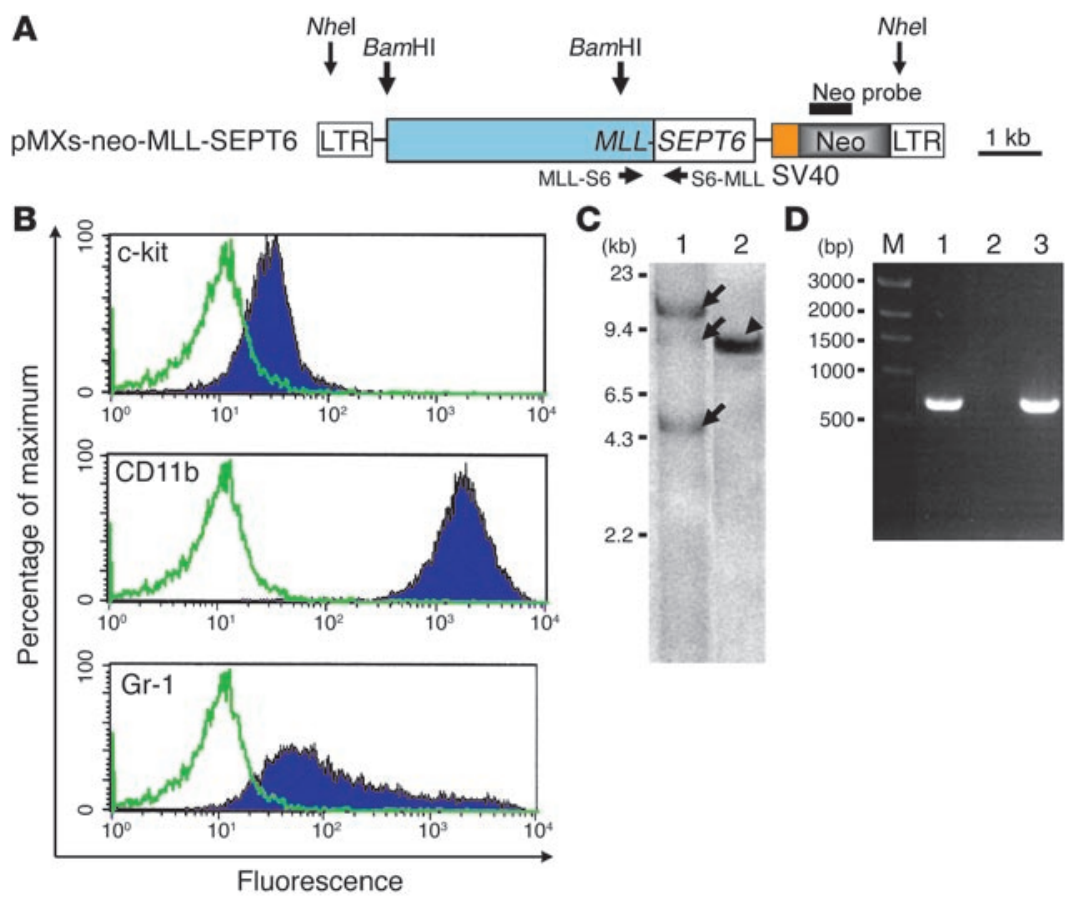


A
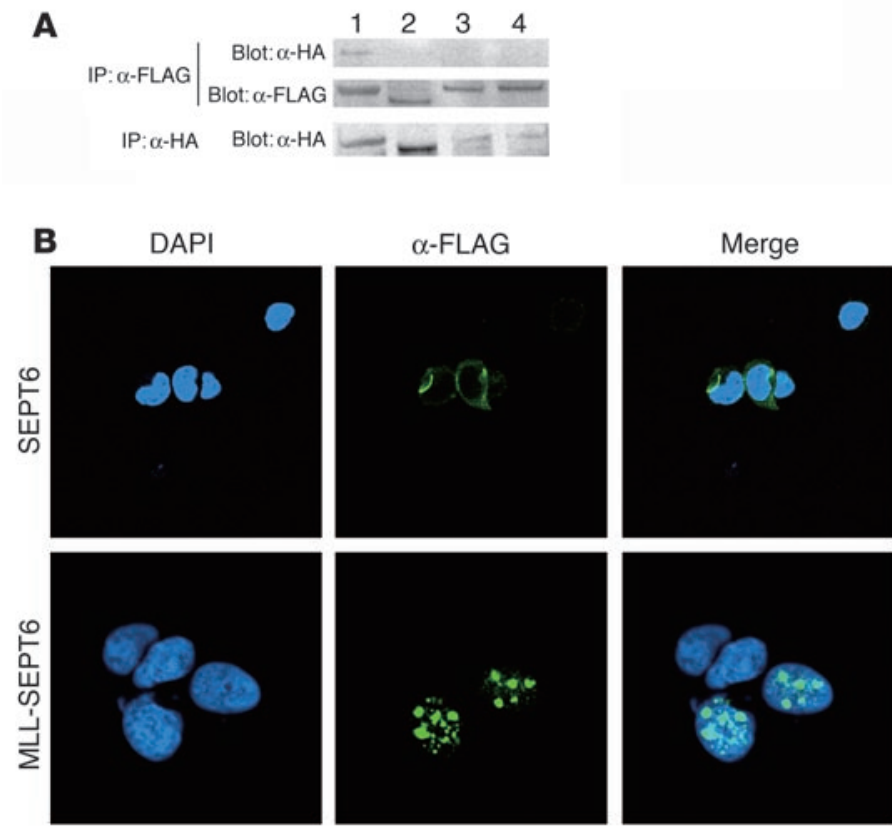

\section{Figure 3}

Oligomerization of MLL-SEPT6 fusion protein through both its GTP-binding domain and its coiled-coil region in the nucleus. 293T cells were cotransfected with equal amounts of FLAG-tagged and HA-tagged constructs $(\mathbf{A})$, and transfected with pMXs-neoSEPT6 or pMXs-neo-MLL-SEPT6 (B). (A) Self-interaction among MLL-SEPT6 fusion proteins or MLL-SEPT6 mutants, analyzed by Western blot analysis after immunoprecipitation. Lysates of 293T cells coexpressing FLAG-tagged and HA-tagged MLL-SEPT6 or its mutants (top and middle) were immunoprecipitated by the anti-FLAG $\mathrm{Ab}$, and lysates of the cells expressing HA-tagged MLL-SEPT 6 or its mutants (bottom) were immunoprecipitated by the anti-HA Ab. AntiFLAG immunoprecipitates were blotted with the anti-HA Ab (top) or the anti-FLAG Ab (middle), while anti-HA immunoprecipitates were blotted with the anti-HA Ab (bottom). Lane 1, MLL-SEPT6; lane 2, MLL-SEPT6 ${ }^{\Delta \text { coil; }}$ lane 3, MLL-SEPT6 ${ }^{\Delta P-l o o p}$; lane 4, MLLSEPT6 ${ }^{556 N}$. (B) Localization of SEPT6 and MLL-SEPT6, analyzed by immunofluorescent confocal microscopy. FITC-conjugated secondary Abs reacting with the anti-FLAG Ab in 293T cells expressing SEPT6 or MLL-SEPT6 visualized their cellular localizations (middle). Nuclei were visualized with DAPI (left), and merged images are displayed (right). Original magnification, $\times 400$. binding domain and a coiled-coil region were required for myeloid immortalization by MLL-SEPT6 in vitro, using various mutants shown in Figure 1A. Our myeloid immortalization assay demonstrated that lack of the GTP-binding domain (MLL-SEPT6 $6^{\triangle \mathrm{GTP}}$ ), a highly conserved P-loop motif within the GTP-binding domain (MLL-SEPT6 ${ }^{\Delta \mathrm{P}-\text { loop}), ~ o r ~ t h e ~ c o i l e d-c o i l ~ r e g i o n ~(M L L-S E P T 6 ~}{ }^{\Delta \text { coil }}$ ) led to failure in colony formation (Figure 1, B and D). Substitution of the critical single amino acid within the P-loop with reduction of GTP-binding activity (MLL-SEPT $6^{\mathrm{S} 56 \mathrm{~N}}$ ), based on previous findings of the well-characterized septin (17), was also found to lead to failure in maintenance of colonies (Figure 1D), and to result in no growth in the liquid culture, demonstrating that MLL-SEPT6 ${ }^{\mathrm{S} 56 \mathrm{~N}}$ also failed to immortalize murine hematopoietic progenitors. These data indicated that the P-loop motif within the GTP-binding domain of SEPT6 was required for MLL-SEPT6-mediated immortalization, suggesting that GTP-binding activity was associated with MLL-SEPT6-mediated immortalization.

To examine whether MLL-SEPT6 homo-oligomerizes through its GTP-binding domain and/or coiled-coil region in the same way as other septins $(18,28)$, mutual-interaction assay of MLLSEPT6 and its mutants using immunoprecipitation/Western blot analysis was first performed as shown in Figure 3. The intact MLL-SEPT6, not any mutants, was coprecipitated (Figure 3A), consistent with the activity of the immortalization in vitro. Furthermore, to investigate whether homo-oligomerization was sufficient for the immortalization, the coiled-coil region of pMXsneo-MLL-SEPT6 was replaced with a mutant ligand-binding domain (LBD) of human estrogen receptor (hER) that induces dimerization specifically in response to 4-hydroxy-tamoxifen (4-OHT) (MLL-SEPT6 ${ }^{\text {coil-ER}}$ ) (29) (Figure 1A). Unexpectedly, MLL-SEPT6 $6^{\Delta \text { coil-ER }}$ (Figure 1B) failed to induce the immortalization in the presence of 4-OHT (Figure 1D), suggesting that homo-oligomerization with proper whole structure is essential for MLL-SEPT6-mediated immortalization. On the other hand, immunofluorescent confocal microscopy of 293T cells transiently expressing MLL-SEPT6 or SEPT6 revealed that MLL-SEPT6 was distributed with a pattern of small speckles or dots in the nucleus (Figure 3B, bottom), while overexpression of SEPT6

\section{Table 1}

Characteristics of morbid mice transplanted with cells immortalized by MLL-SEPT6 in vitro and mice transplanted with hematopoietic progenitors, directly after transduction with MLL-SEPT6, MLL-ENLS, and/or FLT3-ITD

$\begin{array}{lcccccc}\text { Mouse } & \text { Latency (d) } & \text { Liver wt (g) } & \text { Spleen }(\mathbf{g}) & \text { WBC }(\text { cells/ul) } & \text { Hb }(\mathbf{g} / \mathbf{d l}) & \text { Plt }\left(\times 1 \mathbf{1 0}^{4} / \mathbf{\mu l}\right) \\ \text { Immortal. cells }(n=5) & 210 \pm 24 & 3.94 \pm 2.24 & 0.72 \pm 0.23 & 85,000 \pm 75,000 & 8.3 \pm 3.1 & 17 \pm 2.6 \\ \text { PBS }(n=5) & \text { NA } & 1.45 \pm 0.13 & 0.09 \pm 0.01 & 10,000 \pm 2,100 & 14.5 \pm 1.1 & 93 \pm 5.3 \\ \text { MS6/FLT3 }(n=9) & 63 \pm 18 & 1.88 \pm 0.29 & 0.38 \pm 0.07 & 95,000 \pm 70,000 & 9.0 \pm 3.6 & 6.0 \pm 2.5 \\ \text { MS6/GFP }(n=6) & 120 \pm 32 & 2.00 \pm 0.87 & 0.40 \pm 0.15 & 51,000 \pm 18,000 & 7.3 \pm 4.0 & 9.6 \pm 6.4 \\ \text { MES/FLT3 }(n=5)^{A} & 17.6 \pm 1.8 & 2.37 \pm 0.36 & 0.39 \pm 0.09 & 226,200 & 12.6 & 12 \\ \text { MEs/GFP }(n=4) & 92 \pm 9.1 & 2.84 \pm 0.48 & 0.71 \pm 0.17 & 71,000 \pm 22,000 & 5.7 \pm 0.4 & 7.5 \pm 2.1 \\ \text { Mock/FLT3 }(n=5) & \text { NA } & \text { NA } & \text { NA } & 13,000 \pm 4,000 & 13.8 \pm 0.9 & 80 \pm 12 \\ \text { Mock/GFP }(n=4) & \text { NA } & 1.27 \pm 0.11 & 0.07 \pm 0.01 & 13,000 \pm 5,000 & 14.1 \pm 1.2 & 59 \pm 28\end{array}$


alone did not shift its physiological localization in the cytoplasm (Figure 3B, top). Taken together, these data demonstrated that myeloid immortalization in vitro by MLL-SEPT6 requires homooligomerization in the nucleus, probably dimerization of MLL fusion proteins as reported $(11,12)$, through both its GTP-binding domain and its coiled-coil region.

MLL-SEPT6 induces lethal MPD, not acute leukemia, in vivo. We next examined the leukemogenic potential of MLL-SEPT6 in vivo using transplantation of the cells immortalized by MLL-SEPT6 into sublethally irradiated syngeneic mice. Five of 8 mice transplanted with the immortalized cells died with latencies of over 6 months within an observation period of 15 months (Table 1). Morbid mice that received the transplantation exhibited hepatosplenomegaly with leukocytosis, anemia, and thrombocytopenia (Table 1). Notably, histopathologic analysis showed that bone marrow and peripheral blood cells derived from the morbid mice had morphologic and immunophenotypic features of MPD with myeloid hyperplasia consisting predominantly of mature granulocytic elements on FACS analysis, and that these elements infiltrated the spleen and the liver (data not shown). The lethal MPD was also induced by transplantation of murine hematopoietic progenitors transduced with MLL-SEPT6 into lethally irradiated syngeneic mice directly after retroviral infection (described in detail below), and bone marrow cells harvested from these MPD mice grew and expanded dependently on IL-3 in vitro (data not shown). These results differ from findings in the previous reports in which acute leukemias with latencies of 2-6 months were induced by some MLL fusion proteins $(9,10)$, which suggested that fusion of MLL with SEPT6 was not sufficient, and required additional genotoxic stress, to induce acute leukemia.

\section{Figure 4}

Synergistic transformation of murine hematopoietic progenitors by $M L L$ fusion genes and FLT3-ITD in vitro. (A) Schematic representation of the retroviral constructions expressing FLT3-ITD. (B) Transformation assay of the cells immortalized by MLL-SEPT6, after transduction with FLT3-ITD in the pMY-IRESEGFP construct shown in $\mathbf{A}$ in the presence $(+)$ or absence (-) of IL-3. (C) Western blot analysis of proteins extracted from PlatE cells transfected with the constructs shown in $\mathbf{A}$ and each vector alone as a control, after immunoprecipitation using the anti-FLT3 $\mathrm{Ab}$ (lanes 1-4). Each lysate was blotted with the anti-FLT3 Ab. Lane 1, pMY-IRES-EGFP alone; lane 2, pMY-FLT3-ITD-IRES-EGFP; lane 3, pMYpuro alone; lane 4, pMYpuro-FLT3-ITD. (D) Myeloid immortalization assay using the pMYpuro constructs shown in A. The bar graph shows numbers of colonies obtained after each round of replating in methylcellulose (average $\pm \mathrm{SD}$ ). (E) Myeloid transformation assay using the sequential transduction with FLT3-ITD or control (pMYpuro alone) after MLL-SEPT6, $M L L-E N L s$, or mock (pMXs-neo alone) in the presence $(+)$ or absence (-) of IL-3.

D
Secondary genotoxic stress, such as FLT3-ITD, synergistically transforms hematopoietic progenitors transduced with MLL-SEPT6 or MLLENL in vitro. To seek a candidate for additional genotoxic stress required for leukemogenesis by $M L L-S E P T 6$, we first analyzed the transforming activity of MLL-SEPT6-transduced cells with additional expression of FLT3-ITD, using the transformation assay (Figure 4, A and C). The infection efficiencies of FLT3-ITD and vector alone were $0.77 \% \pm 0.31 \%$ and $9.0 \% \pm 0.67 \%$, respectively. The transduction of FLT3-ITD not only enabled the immortalized cells to grow without IL-3 but also turned almost all of the cells $(95.0 \% \pm 1.5 \%)$ positive for GFP 7 days after the deprivation of IL-3, while transduction of the vector alone did not (Figure 4B). These results suggest that FLT3-ITD has the potential to replace the signaling pathways by IL-3 in the cells expressing MLL-SEPT6 in vitro. The transduction of a kinase-active mutant FLT3 with an Asp-835-to-Val mutation (FLT3 ${ }^{\text {D835V }}$ ) also transformed the immortalized cells (data not shown) in the same transformation assay, as it has been reported to transform Ba/F3 cells (30).

On the other hand, the myeloid immortalization assay using FLT3-ITD in pMYpuro could not detect any myeloid immortalization (Figure 4, A, C, and D), which implies that FLT3-ITD alone
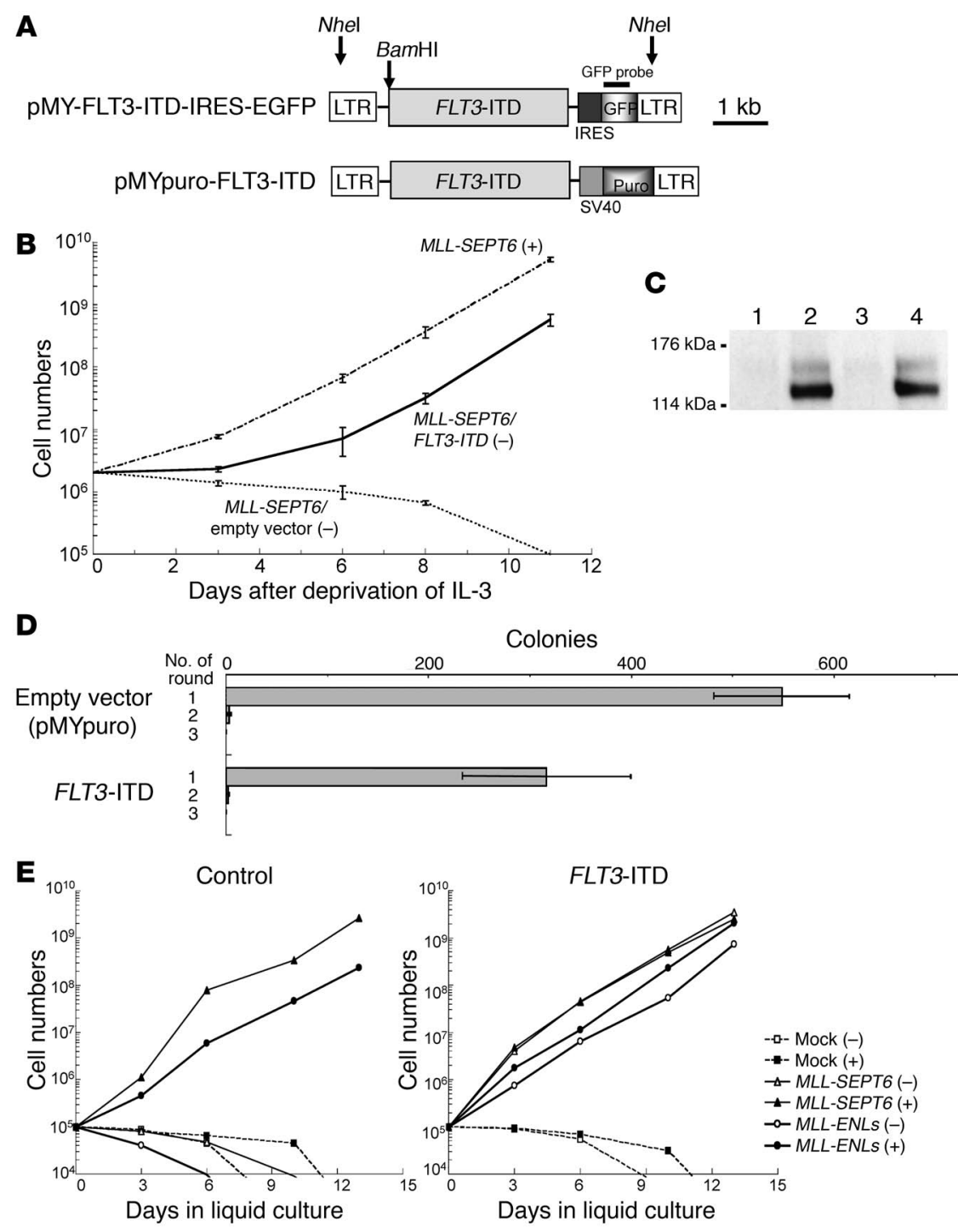

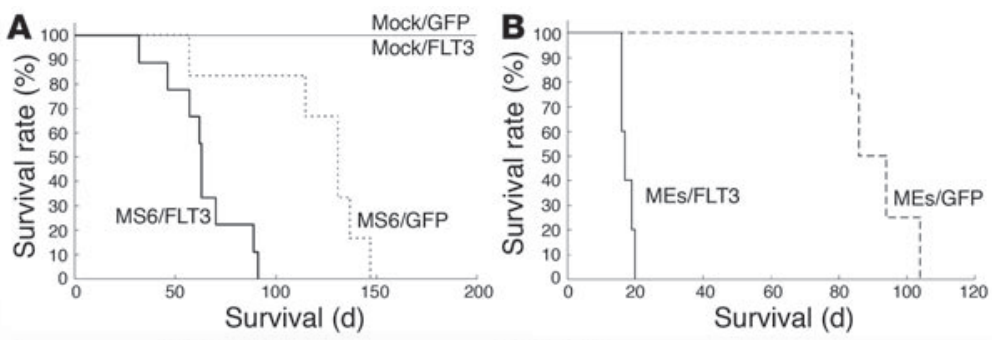

C
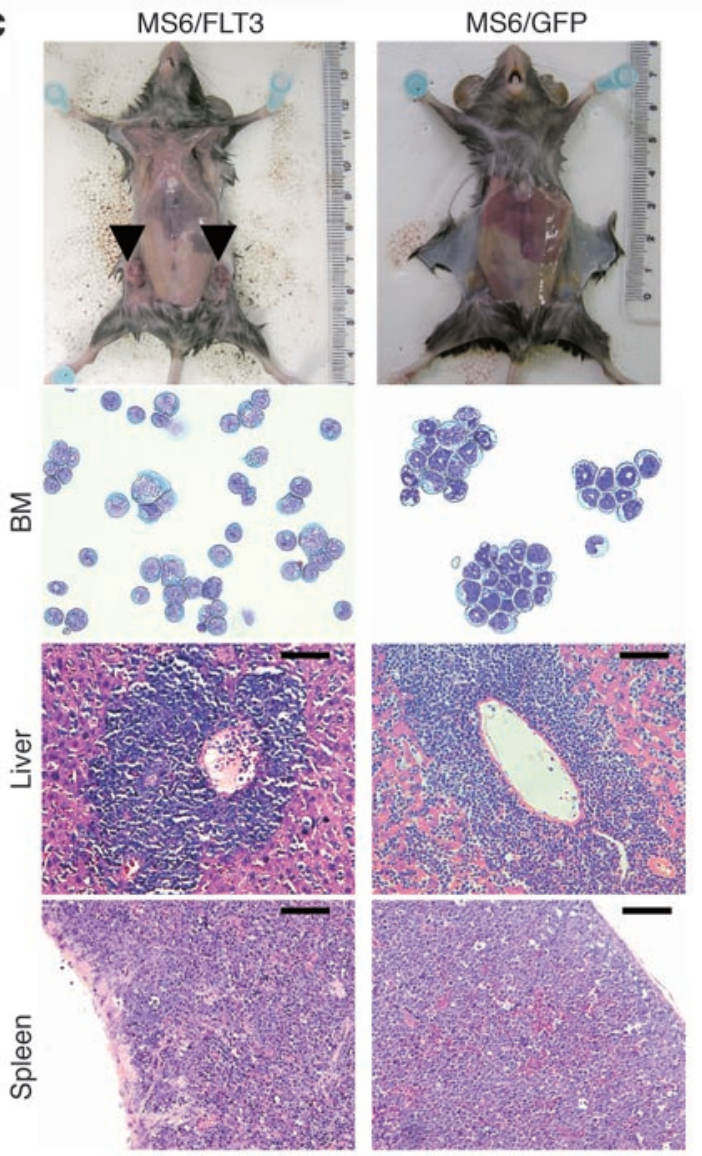

was insufficient for immortalization of murine hematopoietic progenitors in this colony assay or that this assay might be inappropriate to examine the oncogenic proliferative and/or survival advantage, which was considered to be primarily conferred by some oncogenic mutations including FLT3-ITD (31). Furthermore, the synergy of MLL-SEPT6 and FLT3-ITD was examined with myeloid transformation assay using either simultaneous or sequential transduction with these genes. To generalize the synergy in the $M L L$-mediated transformation in vitro, $M L L-E N L s$ was also applied to the same assay. While the simultaneous transduction failed to cause transformation, probably because of the low efficiency of double transduction and limitation of the sensitivity in this in vitro assay (data not shown), the sequential transduction with FLT3-ITD after MLL-SEPT6 or MLL-ENLs, not mock, enabled murine hematopoietic progenitors to grow and expand without IL-3 (Figure 4E). Taken together, these results demonstrated that $M L L$ fusion genes $M L L-S E P T 6$ and $M L L-E N L$ can transform murine hematopoietic progenitors in vitro in concert with additional genotoxic stress, such as FLT3-ITD, which suggests that $M L L$

\section{Figure 5}

Synergistic leukemogenesis induced by $M L L$ fusion genes and FLT3-ITD in vivo. (A and B) Survival curves of mice transplanted with mock/GFP $(n=4), M L L-S E P T 6 / G F P$ (MS6/GFP; $n=6)$, mock/FLT3 $(n=5)$, and MS6/FLT3 $(n=9)(\mathbf{A})$, and with MLL-ENLs/GFP (MEs/GFP; $n=4)$ and MEs/FLT3 $(n=5)(B)$. Mice transplanted with MLL fusion genes in combination with FLT3-ITD showed significantly shorter survival time than those with corresponding $M L L$ fusion genes in combination with GFP $(P<0.05$, log-rank test). (C) Representative macroscopic and histopathologic analysis of morbid mice transplanted with MS6/FLT3 and MS6/GFP, respectively. Arrowheads show lymphadenopathy. BM cells were stained with Wright-Giemsa, and paraffin sections of liver and spleen were stained with $\mathrm{H} \& \mathrm{E}$. Original magnification of BM cells, $\times 200$; scale bars, $200 \mu \mathrm{m}$.

fusion gene and FLT3-ITD may synergistically serve as oncogenes through different pathways.

$M L L$ fusion genes MLL-SEPT6 and MLL-ENL require secondary genotoxic stress, such as FLT3-ITD, to induce acute leukemias in vivo with short latency. To address the synergistic leukemogenic potential of MLL fusion gene and FLT3ITD in vivo, we directly transplanted Ly-5.1 murine hematopoietic progenitors transduced with MLL-SEPT6 and FLT3-ITD (MS6/FLT3) using a combination of each retrovirus into lethally irradiated syngeneic Ly-5.2 mice. The other combinations of each retrovirus mock/GFP (pMXs-neo and pMY-internal ribosomal entry site-enhanced GFP [pMY-IRES-EGFP]), MS6/ GFP, and mock/FLT3 - were also given as controls. In contrast to the MS6/GFP mice, which died with latencies of 57-147 days ( $120 \pm 32$ days), the MS6/FLT3 mice died with significantly shorter latencies of 32-91 days (63 \pm 18 days, $P<0.05$, log-rank test) (Figure 5A and Table 1). The morbid MS6/FLT3 mice exhibited mild hepatomegaly and moderate splenomegaly with various ranges of leukocytosis, anemia, and thrombocytopenia (Table 1). Some of the morbid MS6/FLT3 mice also exhibited remarkable lymphadenopathy (Figure 5C). Histopathologic analysis showed that the majority of bone marrow or peripheral blood cells derived from the morbid MS6/FLT3 mice had morphologic features of immature myelomonocytic blasts, which severely infiltrated the spleen and the liver (Figure 5C). The morphology of the differentiation blockade suggested that the MS6/FLT3 mice developed acute leukemias. On the other hand, the MS6/GFP mice died of MPD that showed features (Figure 5, A and C, and Table 1) similar to those seen in mice transplanted with the cells immortalized by MLL-SEPT6 in vitro, while the mock/FLT3 mice survived without hematological disorders in peripheral blood for an observation period of 6 months (Figure 5A and Table 1). Histopathologic analysis of 1 mock/FLT3 mouse sacrificed 160 days after the transplantation demonstrated neither significant hepatosplenomegaly nor any disorders in the bone marrow, where $20 \%$ of the cells were positive for GFP and hence expressed FLT3-ITD (data not shown).

Cytological analysis showed that almost all of the bone marrow cells $(94.4 \% \pm 9.0 \%)$ derived from the MS6/FLT3 mice originated in donor cells (Ly-5.1), and that the majority of these cells $(85.4 \% \pm 14.9 \%)$ were positive for GFP and thus expressed FLT3ITD (Figure 6). Southern blot analysis of DNA derived from the 

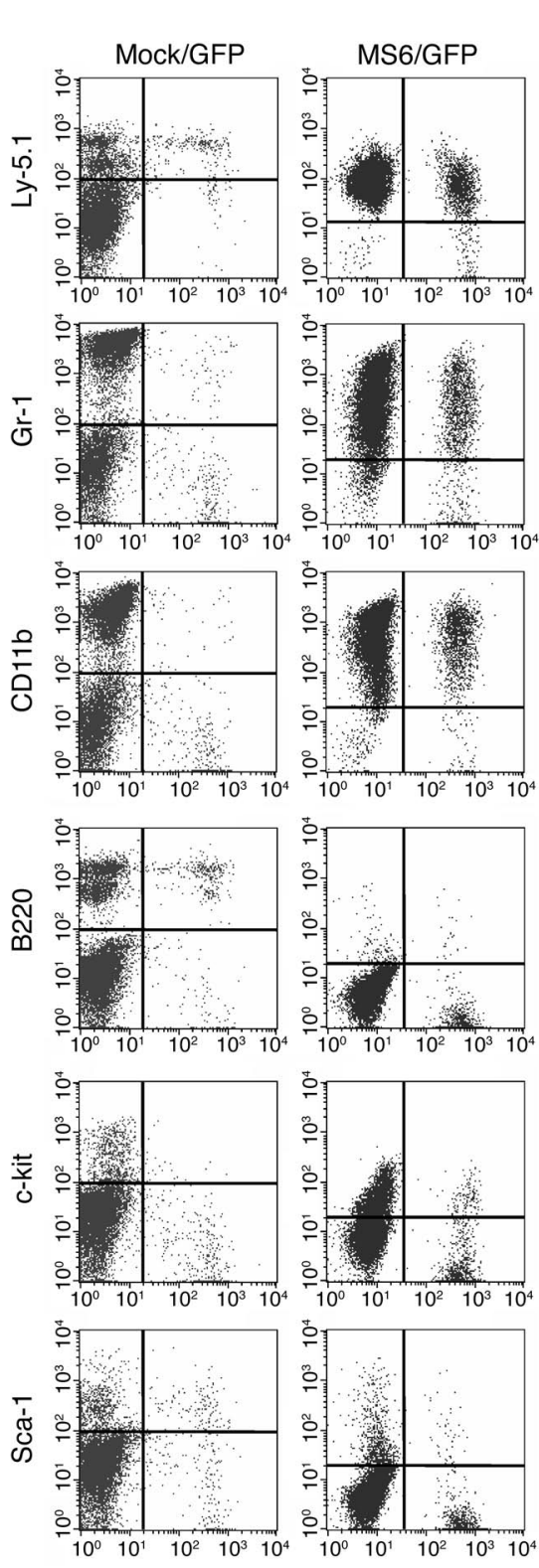
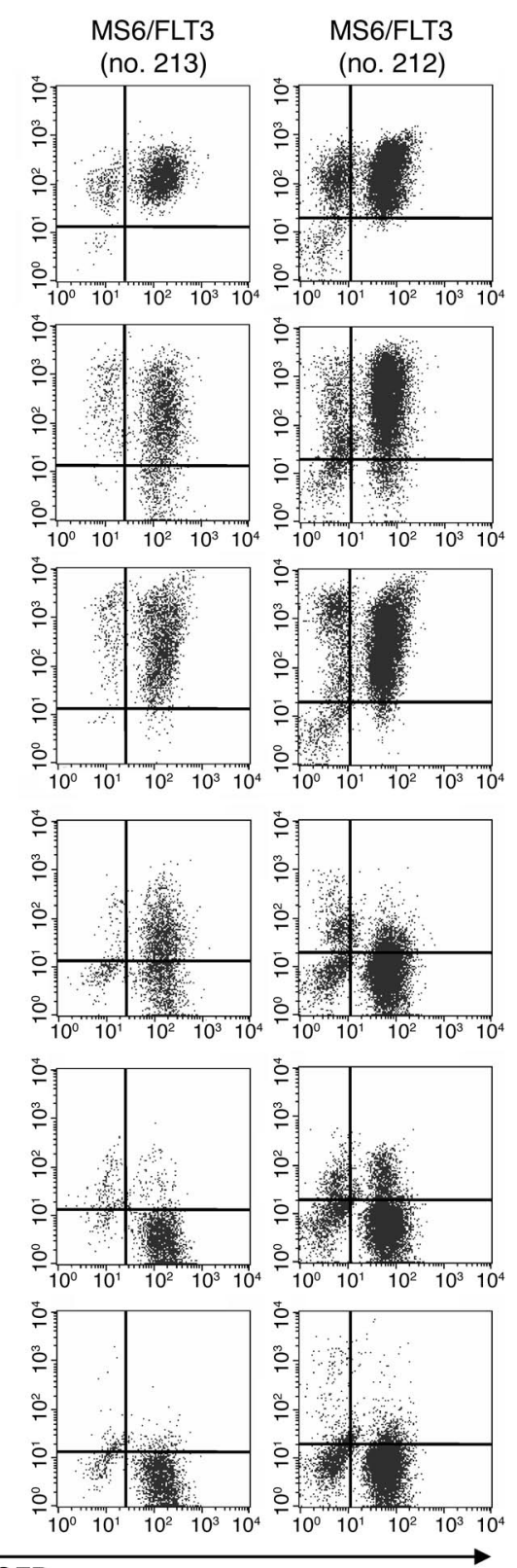

GFP

spleen of the MS6/FLT3 mice demonstrated that intensities of proviral integration bands of MLL-SEPT6 and FLT3-ITD were almost equal, and that oligoclonal bands of proviral integration sites were also present (Figure 7, A and B). In addition, expression of MLL-SEPT6 was detected by RT-PCR analysis of total RNA derived from the spleens (data not shown), indicating both cointegration and coexpression of MLL-SEPT6 and FLT3-ITD in the leukemic cells. Immunophenotyping analysis revealed that these leukemic cells were highly positive for Gr-1 and CD11b (Figure $6)$. Interestingly, the leukemic cells with these myeloid markers sometimes coexpressed B220, but no CD3 (Figure 6 and data not shown), and B220 expression did not always correlate with $\operatorname{IgH}$ gene rearrangements (Figure 7C). For example, $57.0 \%$ and $98.2 \%$ of the GFP-positive leukemic cells derived from mouse no. 213 were positive for B220 and CD11b, respectively (Figure 6), which demonstrates that at least half of these leukemic cells were biphe-

\section{Figure 6}

Immunophenotype of bone marrow cells obtained from representative mice (nos. 213 and 212) transplanted with mock/GFP, MS6/GFP, and MS6/FLT3. The dot plots show each surface antigen labeled with a corresponding PE-conjugated $\mathrm{mAb}$ versus expression of GFP.

notypic, although more direct evidence from 3- or 4-color flow cytometry analysis could not be obtained because of lack of these leukemic cells. These results suggest that MLL-SEPT6 and FLT3-ITD could induce acute myeloid, or sometimes biphenotypic, leukemia with lineage promiscuity (32). Peroxidase stain of these leukemic cells carrying B220 also corroborated myeloid lineage of these blasts (data not shown).

To generalize the synergetic effect in vivo in $M L L$-mediated leukemogenesis, $M L L$-ENLs was also applied to this leukemogenesis assay. MLL-ENLs and FLT3-ITD (MEs/FLT3) could induce AML with significantly shorter latency of $17.6 \pm 1.8$ days $(P<0.05$, log-rank test), while $M L L-E N L s$ (MEs/GFP) induced MPD with latency of $92 \pm 9.1$ days (Table 1, Figure 5B, and data not shown) as previously described (14). Taken together, these data demonstrate that $M L L$ fusion genes $M L L$-SEPT6 and $M L L$ $E N L$ can synergize with secondary genotoxic stress such as FLT3-ITD to induce acute myeloid or biphenotypic leukemias in vivo with short latency as a clinical feature.

\section{Discussion}

We demonstrated that homo-oligomerization of MLL-SEPT6 through its intact GTP-binding domain and coiled-coil region in the nucleus is critical for its oncogenic activity. Intrinsic septins, such as Sept6, tend to polymerize into filaments as heteromeric septin complexes in the cytoplasm at least partially through their coiled-coil regions $(28,33)$, while a recombinant septin, such as Sept2, is able to homopolymerize depending on the binding of GTP, even in the absence of its coiled-coil region (18). Therefore, it is possible that ectopic expression of septin fusion proteins, such as MLL-SEPT6, may result in homo-oligomerization through their GTP-binding domains, and that their coiled-coil regions may contribute to stabilize the homo-oligomerization in the nucleus. Indeed, replacement of the coiled-coil region within MLL-SEPT6 with another inducible dimerization domain, the mutant LBD of hER, did not induce the immortalization in the presence of 4-OHT, which suggests that the coiled-coil region might play some important roles in addition to dimerization (11). It has been revealed that some MLL fusion proteins require oligomerization domains of the partner proteins that normally reside in the cytoplasm and that artificial dimerization of the portion of MLL is sufficient to induce immortalization of hematopoietic progenitors $(11,12)$, while it had already been disclosed that other MLL fusion proteins require the transactivation 

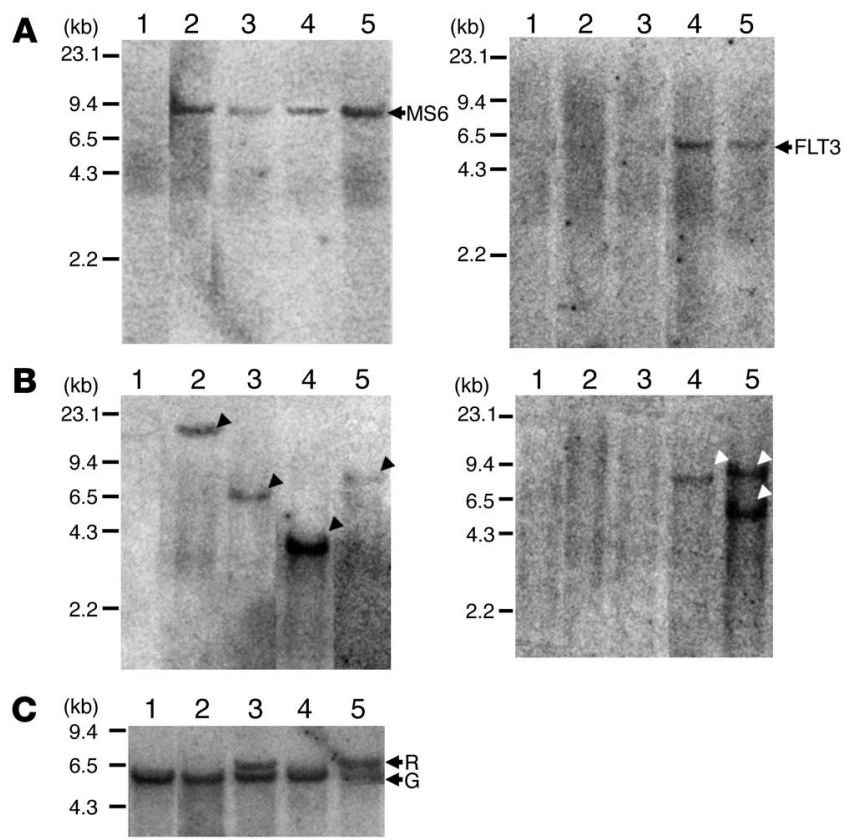

\section{Figure 7}

Southern blot analysis of spleen DNA obtained from representative mice transplanted with mock/GFP, MS6/GFP, and MS6/FLT3. Genomic DNA extracted from each spleen was digested with Nhel (A), BamHI (B), or EcoRI (C) and hybridized with the Neo probe (A and $\mathbf{B}$, left), the GFP probe (A and $\mathbf{B}$, right), or the $\mathrm{J}_{\mathrm{H}}$ probe $(\mathbf{C})$. (A) Each single band of intact proviral DNA, including MLL-SEPT6 (MS6) and FLT3-ITD (FLT3), is indicated by an arrow with each abbreviation. (B) Oligoclonal bands of proviral integration sites of MS6 or FLT3 are indicated by black or white arrowheads, respectively. (C) Germ line $(\mathrm{G})$ or rearrangements $(\mathrm{R})$ of $\mathrm{g} \mathrm{H}$ gene are indicated by an arrow with each abbreviation. Lane 1, mock/GFP mouse; lanes 2 and 3, MS6/ GFP mice; lanes 4 and 5, MS6/FLT3 mice.

domains of the partner proteins that normally reside in the nucleus (9). Our findings that dimerization of MLL-SEPT6 is a prerequisite to develop leukemia also support this notion, but some kinds of simple dimerization of MLL fusion proteins, such as that of MLLSEPT6 $6^{\Delta \text { coil-ER }}$, might be insufficient to induce the immortalization in vitro, as has been reported in the MLL-ENL mutant fused with the mutant LBD of hER (34). On the other hand, the mechanism by which oligomerization of MLL fusion proteins results in transcriptional activation of target genes including $H O X$, some of which were upregulated in our results, is less clear. In addition, Meis 1 was expressed in the cells transduced with mock, as in those with $M L L$ fusion genes, in our myeloid immortalization assay. Replacement of pMXs-neo with pMXs-puro in this immortalization assay reproduced upregulation of Meis1 (data not shown), suggesting that retroviral transduction followed by colony assay itself might accumulate the expression of Meis 1 .

$M L L-S E P T 6$ by itself was able to induce lethal MPD but not to induce acute leukemia in mice, although it did immortalize murine hematopoietic progenitors in vitro. These results differ from previous data $(9,11)$, suggesting 2 possibilities. One possibility is that MLL-SEPT6 itself might possess not so strong an oncogenic potential as other $M L L$ fusion genes examined previously. However, this hypothesis is inconsistent with the strong (i.e., early onset) phenotype of infant AML characteristic of $M L L$ -
SEPT6, which implies that secondary essential genotoxic events that might be evoked by interchromosomal gene rearrangement of $M L L$ and SEPT6, and/or intrinsic unknown genetic instability, are involved in humans (15). It is noteworthy that lethal MPD was recently recapitulated in vivo by Cre-loxP-mediated de novo reciprocal chromosomal translocations bearing Mll-Enl (14). Another possibility is that methodological differences, including the use of different retrovirus vectors, account for the inconsistency with previous data. The promoter activity in the retroviral vectors we used may not be so strong as in the murine stem cell virus vector used in previous reports. But high expression of MLL fusion protein is known to be cytotoxic (9).

We herein provided direct evidence for a multistep leukemogenesis by MLL fusion proteins in vitro and in vivo. It had been suggested that in infant acute leukemia, rearrangements of $M L L$ arise in utero and result in leukemia after birth (15). The phenotype of Mll-AF9 knock-in mice that develop overt leukemia with long latency after birth and do not acquire sufficient oncogenic potential prenatally or perinatally $(13,35)$ supports the multistep leukemogenesis. Other experimental models in vivo $(9,10)$ did require a longer latency than expected from clinical features of human infant leukemia, which also implies that some secondary genotoxic events are essential for leukemogenesis by MLL fusion proteins. Vigorous investigations using DNA microarray revealed that FLT3 is associated with $M L L$-rearranged leukemia (36), and there has been an interesting report that $M L L-E N L$ induced acute multilineage leukemia in vivo only in combination with an activated receptor tyrosine kinase in a chicken model (37). The mutations of FLT3 with constitutive tyrosine kinase activity are classified into FLT3-ITD or mutations within the activation loop (FLT3-mut), such as FLT3 ${ }^{\mathrm{D} 835 \mathrm{~V}}$. FLT3-ITDs are associated with adult AML with poor prognosis (31), while FLT3-muts are found frequently in infant acute lymphoid leukemia with $M L L$ rearrangements (38). Therefore, even though infant AML with FLT3-ITD has yet to be reported in studies of the limited number of cases of pediatric AML with 11q23 translocations (39), FLT3-ITD was assigned to a secondary genotoxic event in our experimental model system by both the clinical feature of infant AML with MLL-SEPT6 and the presence of the myeloid and biphenotypic leukemic cell lines MOLM-13 and MV4;11, which harbor FLT3-ITD as well as rearrangements of MLL (38).

Recent reports demonstrated that FLT3-ITD induces not only MPD by itself (40), but also acute leukemia in concert with $P M L-R A R \alpha$ which alone causes MPD (41). Indeed, additional transduction of FLT3-ITD synergistically led to both in vitro transformation of MLL-SEPT6-transduced cells and in vivo development of acute leukemia with shorter latency by MLL-SEPT6, although the transduction of FLT3-ITD alone was not sufficient to induce lethal MPD with short latency in our experimental model, where we did not dare to intentionally enhance retroviral transduction since the enhancement could lead to FLT3-ITD-induced MPD (R. Ono, H. Nakajima, T. Kitamura, and T. Nosaka, unpublished observations) as reported previously (40). Taken together with the findings of stability of $M L L$ fusion genes (5) and instability of FLT3-ITD in relapse samples (42), our results of the synergistic effects of MLL-SEPT6 and FLT3-ITD on leukemogenesis indicate that FLT3-ITD can confer necessary and sufficient secondary genotoxicity on leukemogenesis by MLL-SEPT6. The bone marrow cells harvested from the mice developing MPD induced by direct transduction with MLL-SEPT6 grew and expanded dependently on IL-3 in vitro as reported (43), suggesting that induction of 
IL-3 independence by FLT3-ITD might be at least partly responsible for the leukemogenesis by MLL-SEPT6 in concert with FLT3ITD. Furthermore, since $M L L-E N L$, which requires no dimerization but a transactivation domain within ENL for its oncogenic activity (9), also induces transformation of murine hematopoietic progenitors in vitro and AML in vivo, in concert with FLT3-ITD, it is likely that MLL fusion proteins serve primarily to impair differentiation and/or to enhance self-renewal of hematopoietic progenitors and that FLT3-ITD confers the oncogenic proliferative and/or survival advantage (31). Interestingly, although FLT3-ITD is strongly associated with myeloid lineage, acute leukemia induced by MLL-SEPT6 in concert with FLT3-ITD possessed not only myeloid but also biphenotypic lineage markers, as evidenced by the combination of $M L L-E N L$ and an activated tyrosine kinase (37). This suggests that the multilineage property of acute leukemia with some rearrangements of $M L L$ may require mutations such as constitutively active tyrosine kinases. Finally, these 2 -step model systems will provide novel insights into the multistep leukemogenesis of acute myeloid and biphenotypic leukemia with 11q23 translocations.

\section{Methods}

Retroviral constructs. The 5'-MLL fragment covering MLL exons 1-7 (a kind gift from H. Hirai, The University of Tokyo), and the full length of SEPT6 type I (16), were inserted into PMXs-neo to generate PMXs-neo-5'-MLL and pMXs-neo-SEPT6, respectively. Several fragments containing portions of SEPT6, ENL (a kind gift from M. Seto, Aichi Cancer Center Research Institute, Aichi, Japan), or the mutant LBD of hER (29), produced with PCR, were cloned into PMXs-neo-5'-MLL for various PMXs-neo constructs. Each fragment inserted into pMXs-neo was fused with a FLAG or HA epitope tag at the C-terminus. A fragment of FLT3-ITD (30) was also inserted upstream of the IRES-EGFP cassette of PMY-IRES-EGFP to generate pMYFLT3-ITD-IRES-EGFP, and the IRES-EGFP cassette was replaced with the puromycin cassette of pMXs-puro (27) to generate PMYpuro-FLT3-ITD.

Transfection and retrovirus production. Transfection was carried out using FuGENE 6 (Roche Diagnostics Corp.) according to the manufacturer's recommendations. Retroviruses were harvested 48 hours after PlatE cells were transfected with retroviral constructs, as previously described (30). Appropriate expression of the transgenes in PlatE cells was confirmed by Western blot analysis.

Production of a polyclonal Ab against MLL. The anti-MLL Ab against an oligopeptide within the portion of MLL retained in MLL fusion proteins (44) was manufactured in rabbits, using standard methods.

Immunoprecipitation and Western blot analysis. PlatE or 293T cells transfected with various constructs, or the MLL-SEPT6-transduced cells, were harvested with the lysis buffer (500 mM NaCl, $20 \mathrm{mM}$ HEPES [pH 7.4], $0.5 \mathrm{mM}$ EDTA, $2 \mathrm{mM}$ DTT, $0.2 \% \mathrm{NP}-40$ ), supplemented with protease inhibitor cocktail (Sigma-Aldrich), and left on ice for 3 minutes. In mutual-interaction assay of MLL-SEPT6 fusion protein and its mutants, cell lysates were immediately diluted only for $\mathrm{NaCl}$ on ice, resulting in $150 \mathrm{mM}$ of $\mathrm{NaCl}$. Cell lysates were subjected to immunoprecipitation with the anti-MLL, monoclonal antiHA (12CA5) (Roche Diagnostics Corp.), monoclonal anti-FLAG (M2), or polyclonal anti-FLT3 (C-20) (Santa Cruz Biotechnology Inc.) Ab as previously described (45), except that samples were washed with diluted lysis buffer (150 mM NaCl, 20 mM HEPES [pH 7.4], 0.5 mM EDTA, 2 mM DTT, $0.2 \%$ NP-40) supplemented with protease inhibitor cocktail in the mutual-interaction assay. Cell lysates transfected with pMXs-neo-SEPT6 were directly mixed with an equal volume of $2 \times$ SDS sample buffer ( $125 \mathrm{mM}$ Tris- $\mathrm{HCl}$ [pH 6.8], $4 \%$ SDS, $20 \%$ glycerol, $10 \%$ 2-mercaptoethanol, $0.04 \%$ bromophenol blue), and boiled for 5 minutes. Western blot analysis of the immunoprecipitate of each sample and the samples obtained from the cells transfected with pMXs-
neo-SEPT6 was done using the anti-FLAG, anti-HA, anti-FLT3, or polyclonal anti-SEPT6 (a kind gift from M. Kinoshita, Kyoto University, Kyoto, Japan) (33) Ab to probe membranes as previously described (45).

Myeloid immortalization assays and transformation assays of hematopoietic progenitors in vitro. The oncogenic potential of MLL-SEPT6 or MLL-ENLs fusion protein and/or FLT3-ITD in vitro was analyzed in modified myeloid immortalization assays of murine hematopoietic progenitors, as previously described (43). In brief, hematopoietic progenitors were harvested from 6- to 10-week-old C57BL/6 mice 4 days after i.p. administration of 150 $\mathrm{mg} / \mathrm{kg}$ 5-fluorouracil (5-FU), and cultured overnight in aMEM supplemented with $20 \%$ FCS and $50 \mathrm{ng} / \mathrm{ml}$ each of mouse SCF, human IL-6, human FLT3 ligand (R\&D Systems Inc.), and human thrombopoietin (Kirin Brewery Co.). The prestimulated cells were infected for 60 hours with the retroviruses harboring various MLL fusion genes in PMXs-neo, FLT3ITD in pMYpuro, or empty vector as control, in the aMEM supplemented with the same FCS and cytokines, using 6-well dishes coated according to the manufacturer's recommendations with RetroNectin (Takara Bio Inc.). Coinfection in the myeloid transformation assay, using simultaneous transduction, was done with mixtures of each retrovirus produced independently in equal mole ratio of each insert between long-terminal repeats in each retroviral construct. Infected cells ( $10^{5}$ except for $10^{6}$ in coinfection) were then plated in $1.1 \mathrm{ml}$ of $1 \%$ methylcellulose medium (StemCell Technologies) supplemented with $50 \mathrm{ng} / \mathrm{ml}$ of SCF and $10 \mathrm{ng} / \mathrm{ml}$ each of mouse IL-3, IL-6, and mouse GM-CSF (R\&D Systems Inc.) in the presence of the appropriate drug or drugs (i.e., $1 \mathrm{mg} / \mathrm{ml}$ of $\mathrm{G} 418,1 \mu \mathrm{g} / \mathrm{ml}$ of puromycin, or a mixture of both). After culture for 1 week, colonies were counted, and single-cell suspensions $\left(10^{4}\right.$ cells $)$ of drug-resistant colonies were subsequently replated under identical conditions without any drug. Every 1 week, replating was repeated in the same way. Experiments with the pMXs-neo construct harboring fusion with the mutant LBD of hER were performed in the presence of either $1 \mu \mathrm{M}$ of 4-OHT or vehicle control (ethanol). The immortalized cells were generated by seeding of cells from the third round of methylcellulose cultures in RPMI 1640 medium supplemented with $20 \%$ FCS and $10 \mathrm{ng} / \mathrm{ml}$ of IL-3. In myeloid transformation assays using sequential transduction, the G418-resistant cells harvested at the first round were secondarily infected with retroviruses harboring or not harboring FLT3-ITD in pMYpuro using the same protocol as in the first infections, and replated under identical conditions in the presence of puromycin. After 1 week, these transduced cells were cultured in RPMI 1640 medium supplemented with $20 \%$ FCS and SCF $(20 \mathrm{ng} / \mathrm{ml}$, only for the first 1 week) in the presence or absence of IL-3 (10 ng/ml), and viable cell numbers were counted periodically after standard trypan blue staining.

Tumorigenicity and leukemogenesis assays in vivo. For tumorigenicity assays of the cells immortalized by MLL-SEPT6, $10^{6}$ of the immortalized cells, or PBS as negative control, were injected i.v. into 8- to 12-week-old C57BL/6 (Ly-5.2) mice, which had been administered a sublethal dose of 5.25 Gy total-body $\gamma$-irradiation $\left({ }^{135} \mathrm{Cs}\right)$. For assays of leukemogenesis by $M L L-S E P T 6$ or $M L L$ ENLs in concert with FLT3-ITD, hematopoietic progenitors harvested after 5-FU treatment of Ly-5.1 C57BL/6 mice were prestimulated in the same way as in the myeloid immortalization assays. Using 6-well dishes coated with RetroNectin, the prestimulated progenitors were infected with mixtures of retroviruses under identical conditions to those for myeloid immortalization assays. These mixtures consisted of combinations of retroviruses harboring $M L L$-SEPT6, MLL-ENLs, or no insert in PMXs-neo, and FLT3-ITD or no insert in PMY-IRES-EGFP, as in the coinfection in the myeloid immortalization assay. Infected Ly-5.1 cells $\left(10^{5}\right)$ were injected i.v. together with a radioprotective dose of $2 \times 10^{5}$ Ly-5.2 cells into Ly-5.2 mice, which had been administered a lethal dose of 9.5 Gy total-body $\gamma$-irradiation. In both assays, morbid mice were euthanized, and their tissue samples, such as peripheral blood, bone marrow, liver, and spleen, were 
analyzed. Circulating blood cells were counted, and tissues were fixed in $10 \%$ buffered formalin, embedded in paraffin, sectioned, and stained with H\&E, as previously described (46). Probabilities of murine overall survival were estimated using the Kaplan-Meier method and compared using the log-rank test. All animal studies were approved by the Animal Care Committee of the Institute of Medical Science, The University of Tokyo.

Transformation assays of the cells immortalized by MLL-SEPT6 in vitro. Cells immortalized by MLL-SEPT6, which grew dependently on IL-3, were infected with retroviruses harboring or not harboring FLT3-ITD in PMY-IRESEGFP, as previously described (45). To determine whether the infected cells were transformed, these cells were deprived of IL-3 24 hours after the infection, and viable cell numbers were counted periodically after standard trypan blue staining.

Morphological and FACS analysis. Cytospin preparations of the cells immortalized by MLL-SEPT6 and of bone marrow cells, and blood smears, were stained with Wright-Giemsa or peroxidase stain to assess cell morphology. Immunophenotyping and assessment of GFP-positivity were done with a FACSCalibur (BD Biosciences) as previously described (46).

Southern blot analysis. Genomic DNA was extracted from tissues or the immortalized cells and analyzed with a 352 -bp GFP probe spanning nucleotides 63-414 (Figure 4A), the Neo probe (46) (Figure 2A), or the $\mathrm{J}_{\mathrm{H}}$ probe (a kind gift from C. Coleclough, St. Jude Children's Research Hospital, Memphis, Tennessee, USA) (47) as previously described (46).

RT-PCR. Total RNA was extracted from tissues, the immortalized cells, or a murine pro- $\mathrm{B}$ cell line, $\mathrm{Ba} / \mathrm{F} 3$, and reverse-transcribed to $\mathrm{CDNA}$ with random hexamers as previously described (16). Conditions, reagents for PCR, and the primers specific for $\beta_{2} \mathrm{~m}$ were as previously described (46). To detect transcripts of MLL-SEPT6, Hox a7, Hox a9, and Meis1, PCR amplification was run for 30 cycles using primers as follows: MLL-S6, 5'-GTGAAGAACGTGGTGGACTCTAG-3', and S6-MLL, 5'-TGGCTGGCTCCCCTTCGAA3'; Hoxa7S, 5'-TGCGCCTCCTACGACCAAAACATC-3', and Hoxa7AS, 5'-CTCTTTCTTCCACTTCATGCGCCGA-3'; Hoxa9S, 5' -TGGCATTA-
AACCTGAACCGCTCTCG-3', and Hoxa9AS, 5'-CTTCATTTTCATCCTGCGGT-TCTGGAAC-3'; M1S, 5'-GCATGGGTTCCTCGGTCAATGAC-3', and M1AS, 5'-CATGGTCTCTATTCC-AAGAGGGCTG-3'.

Immunostaining. Immunostaining of $293 \mathrm{~T}$ cells was done and viewed with a FluoView FV300 confocal microscope (Olympus Corp.), as previously described (48).

\section{Acknowledgments}

We thank Hisamaru Hirai for the plasmid harboring a fragment of $M L L$, Masao Seto for the plasmid harboring MLL-ENL, Makoto Kinoshita for the anti-SEPT6 Ab, and Christopher Coleclough for the $\mathrm{J}_{\mathrm{H}}$ probe. We are also grateful to Mineo Takagi (Shinshu University, Nagano, Japan) and Kouichi Ariyoshi (Yamaguchi University, Yamaguchi, Japan) for the protocol of transplantation in mice, Yukinori Minoshima for immunostaining and confocal microscopy, Ai Hishiya for cultures, Fumi Shibata for a pMY-FLT3-ITD-IRESEGFP construct, Mao Sakita-Ishikawa and Ai Hishiya for maintaining the mice, and Mariko Ohara for language assistance. The Division of Hematopoietic Factors is supported in part by the Chugai Pharmaceutical Company Ltd. R. Ono is supported by a research fellowship from the Japan Society for the Promotion of Science. This work was supported in part by Grants-in-Aid from the Ministry of Education, Culture, Sports, Science and Technology in Japan.

Received for publication July 15, 2004, and accepted in revised form January 18, 2005.

Address correspondence to: Tetsuya Nosaka, Division of Hematopoietic Factors, The Institute of Medical Science, The University of Tokyo, 4-6-1 Shirokanedai, Minato-ku, Tokyo 1088639, Japan. Phone: 81-3-5449-5399; Fax: 81-3-5449-5453; E-mail: tenosaka@ims.u-tokyo.ac.jp.
1. Look, A.T. 1997. Oncogenic transcription factors in the human acute leukemias. Science. 278:1059-1064.

2. Rowley, J.D. 1998. The critical role of chromosome translocations in human leukemias. Annu. Rev. Genet. 32:495-519.

3. Tkachuk, D.C., Kohler, S., and Cleary, M.L. 1992. Involvement of a homolog of Drosophila trithorax by $11 \mathrm{q} 23$ chromosomal translocations in acute leukemias. Cell. 71:691-700

4. Gu, Y., et al. 1992 . The $t(4 ; 11)$ chromosome translocation of human acute leukemias fuses the $A L L-1$ gene, related to Drosophila trithorax, to the $A F-4$ gene. Cell. 71:701-708.

5. Hayashi, Y. 2000. The molecular genetics of recurring chromosome abnormalities in acute myeloid leukemia. Semin. Hematol. 37:368-380.

6. Milne, T.A., et al. 2002. MLL targets SET domain methyltransferase activity to Hox gene promoters. Mol. Cell. 10:1107-1117.

7. Nakamura, T., et al. 2002. ALL-1 is a histone methyltransferase that assembles a supercomplex of proteins involved in transcriptional regulation. Mol. Cell. 10:1119-1128.

8. Hsieh, J.J., Cheng, E.H., and Korsmeyer, S.J. 2003 Taspase 1: a threonine aspartase required for cleavage of MLL and proper HOX gene expression. Cell. 115:293-303

9. Ayton, P.M., and Cleary, M.L. 2001. Molecular mechanism of leukemogenesis mediated by MLL fusion proteins. Oncogene. 20:5695-5707.

10. Daser, A., and Rabbitts, T.H. 2004. Extending the repertoire of the mixed-lineage leukemia gene $M L L$ in leukemogenesis. Genes Dev. 18:965-974.

11. So, C.W., Lin, M., Ayton, P.M., Chen, E.H., and Cleary, M.L. 2003. Dimerization contributes to oncogenic activation of MLL chimeras in acute leukemias. Cancer Cell. 4:99-110.

12. Martin, M.E., et al. 2003. Dimerization of MLL fusion proteins immortalizes hematopoietic cells. Cancer Cell. 4:197-207.

13. Corral, J., et al. 1996. An Mll-AF9 fusion gene made by homologous recombination causes acute leukemia in chimeric mice: a method to create fusion oncogenes. Cell. 85:853-861.

14. Forster, A., et al. 2003. Engineering de novo reciprocal chromosomal translocations associated with Mll to replicate primary events of human cancer. Cancer Cell. 3:449-458.

15. Greaves, M.F., and Wiemels, J. 2003. Origins of chromosome translocations in childhood leukaemia. Nat. Rev. Cancer. 3:639-649.

16. Ono, R., et al. 2002. SEPTIN6, a human homolog to mouse Septin6, is fused to $M L L$ in infant acute myeloid leukemia with complex chromosomal abnormalities involving $11 \mathrm{q} 23$ and $\mathrm{Xq} 24$. Cancer Res. 62:333-337.

17. Kinoshita, M., et al. 1997. Nedd5, a mammalian septin, is a novel cytoskeletal component interacting with actin-based structures. Genes Dev. 11:1535-1547

18. Mendoza, M., Hyman, A.A., and Glotzer, M. 2002. GTP binding induces filament assembly of a recombinant septin. Curr. Biol. 12:1858-1863.

19. Megonigal, M.D., et al. 1998. t(11;22)(q23;q11.2) in acute myeloid leukemia of infant twins fuses $M L L$ with $h C D C r e l$, a cell division cycle gene in the genomic region of deletion in DiGeorge and velocardiofacial syndromes. Proc. Natl. Acad. Sci. U. S. A. 95:6413-6418.

20. Taki, T., et al. 1999. AF17q25, a putative septin family gene, fuses the $M L L$ gene in acute myeloid leukemia with $\mathrm{t}(11 ; 17)(\mathrm{q} 23 ; \mathrm{q} 25)$. Cancer Res. 59:4261-4265.

21. Osaka, M., Rowley, J.D., and Zeleznik-Le, N.J. 1999. MSF (MLL septin-like fusion), a fusion partner gene of $M L L$, in a therapy-related acute myeloid leukemia with a t(11;17)(q23;q25). Proc. Natl. Acad. Sci.U. S. A. 96:6428-6433.

22. Kojima, K., et al. 2004. FLJ10849, a septin family gene, fuses $M L L$ in a novel leukemia cell line CNLBC1 derived from chronic neutrophilic leukemia in transformation with $\mathrm{t}(4 ; 11)(\mathrm{q} 21 ; \mathrm{q} 23)$. Leukemia. 18:998-1005.

23. Borkhardt, A., et al. 2001. An ins(X;11)(q24;q23) fuses the MLL and the Septin 6/KIAA0128 gene in an infant with AML-M2. Genes Chromosomes Cancer. 32:82-88.

24. Slater, D.J., et al. 2002. MLL-SEPTIN6 fusion recurs in novel translocation of chromosomes $3, \mathrm{X}$, and 11 in infant acute myelomonocytic leukaemia and in $\mathrm{t}(\mathrm{X} ; 11)$ in infant acute myeloid leukaemia, and $M L L$ genomic breakpoint in complex MLL-SEPTIN6 rearrangement is a DNA topoisomerase II cleavage site. Oncogene. 21:4706-4714.

25. Kim, H.J., et al. 2003. MLL/SEPTIN6 chimeric transcript from inv ins $(X ; 11)(q 24 ; q 23 q 13)$ in acute monocytic leukemia: report of a case and review of the literature. Genes Chromosomes Cancer. 38:8-12.

26. Fu, J.F., Liang, D.C., Yang, C.P., Hsu, J.J., and Shih, L.Y. 2003. Molecular analysis of $t(X ; 11)(q 24 ; q 23)$ in an infant with AML-M4. Genes Chromosomes Cancer. 38:253-259.

27. Kitamura, T., et al. 2003. Retrovirus-mediated gene transfer and expression cloning: powerful tools in functional genomics. Exp. Hematol. 31:1007-1014.

28. Sheffield, P.J., et al. 2003. Borg/septin interac- 
tions and the assembly of mammalian septin heterodimers, trimers, and filaments. J. Biol. Chem. 278:3483-3488.

29. Walkley, C.R., et al. 2004. Identification of the molecular requirements for an RAR alpha-mediated cell cycle arrest during granulocytic differentiation. Blood. 103:1286-1295.

30. Murata, K., et al. 2003. Selective cytotoxic mechanism of GTP-14564, a novel tyrosine kinase inhibitor in leukemia cells expressing a constitutively active Fms-like tyrosine kinase 3 (FLT3). J. Biol. Chem. 278:2892-2898.

31. Gilliland, D.G., and Griffin, J.D. 2002. The roles of FLT3 in hematopoiesis and leukemia. Blood. 100:1532-1542.

32. Greaves, M.F., Chan, L.C., Furley, A.J., Watt, S.M., and Molgaard, H.V. 1986. Lineage promiscuity in hemopoietic differentiation and leukemia. Blood. 67:1-11.

33. Kinoshita, M., Field, C.M., Coughlin, M.L., Straight, A.F., and Mitchison, T.J. 2002. Self- and actin-templated assembly of Mammalian septins. Dev. Cell. 3:791-802.

34. Ayton, P.M., and Cleary, M.L. 2003. Transformation of myeloid progenitors by MLL oncoproteins is dependent on Hoxa7 and Hoxa9. Genes Dev. 18:2298-2307.
35. Johnson, J.J., et al. 2003. Prenatal and postnatal myeloid cells demonstrate stepwise progression in the pathogenesis of $M L L$ fusion gene leukemia. Blood. 101:3229-3235.

36. Armstrong, S.A., et al. 2002. $M L L$ translocations specify a distinct gene expression profile that distinguishes a unique leukemia. Nat. Genet. 30:41-47.

37. Schulte, C.E., et al. 2002. MLL-ENL cooperates with SCF to transform primary avian multipotent cells. EMBO J. 21:4297-4306.

38. Taketani, T., et al. 2004. FLT3 mutations in the activation loop of tyrosine kinase domain are frequently found in infant ALL with $M L L$ rearrangements and pediatric ALL with hyperdiploidy. Blood. 103:1085-1088.

39. Zwaan, C.M., et al. 2003. FLT3 internal tandem duplication in 234 children with acute myeloid leukemia: prognostic significance and relation to cellular drug resistance. Blood. 102:2387-2394.

40. Kelly, L.M., et al. 2002. FLT3 internal tandem duplication mutations associated with human acute myeloid leukemias induce myeloproliferative disease in a murine bone marrow transplant model. Blood. 99:310-318.

41. Kelly, L.M., et al. 2002. PML/RARalpha and FLT3ITD induce an APL-like disease in a mouse model. Proc. Natl. Acad. Sci. U. S. A. 99:8283-8288.
42. Kottaridis, P.D., et al. 2002. Studies of FLT3 mutations in paired presentation and relapse samples from patients with acute myeloid leukemia: implications for the role of FLT3 mutations in leukemogenesis, minimal residual disease detection, and possible therapy with FLT3 inhibitors. Blood. 100:2393-2398.

43. Lavau, C., Szilvassy, S.J., Slany, R., and Cleary, M.L. 1997. Immortalization and leukemic transformation of a myelomonocytic precursor by retrovirally transduced HRX-ENL. EMBO J. 16:4226-4237.

44. Butler, L.H., Slany, R., Cui, X., Cleary, M.L., and Mason, D.Y. 1997. The HRX proto-oncogene product is widely expressed in human tissues and localizes to nuclear structures. Blood. 89:3361-3370.

45. Nosaka, T., et al. 1999. STAT5 as a molecular regulator of proliferation, differentiation and apoptosis in hematopoietic cells. EMBOJ. 18:4754-4765.

46. Nosaka, T., et al. 2003. Mammalian twisted gastrulation is essential for skeleto-lymphogenesis. Mol. Cell. Biol. 23:2969-2980.

47. Coleclough, C., Perry, R.P., Karjalainen, K., and Weigert, M. 1981. Aberrant rearrangements contribute significantly to the allelic exclusion of immunoglobulin gene expression. Nature. 290:372-378.

48. Minoshima, Y., et al. 2003. Phosphorylation by aurora B converts MgcRacGAP to a RhoGAP during cytokinesis. Dev. Cell. 4:549-560. 